\title{
Label-free 3D Imaging of Development of Cell Patterns in Drosophila melanogaster Wing Imaginal Disc
}

\author{
G. Rago, ${ }^{*}$ F. Marty, ${ }^{*}$ J.P.R. Day,* R. Heeren, ${ }^{*}$ K. Basler, ${ }^{* *}$ E. Brunner, ${ }^{* * *}$ M. Bonn* \\ * FOM Institute AMOLF, Science Park 104, 1098XG Amsterdam, The Netherlands. \\ ** Institute of Molecular Life Sciences, University of Zurich. Winterthurerstrasse 190, CH-8057 \\ Zurich, Switzerland. \\ *** Q-MOP, University of Zurich, Winterthurerstrasse 190, CH-8057 Zurich, Switzerland.
}

At the basis of developmental biology lies the understanding of how the development of size and shape of organs is regulated in the growth process. Evidence has been presented for a strong connection in embryogenesis between the appearance of clusters of specific cells and the proliferation of these cells resulting in the growth of a specific tissue. Despite extensive efforts to understand how sub-compartments are generated during organ development, little is known on the role that the development of cell patterns and the molecular composition of cells from different compartments play in the proliferation process. Such compartments in the wing imaginal disc of Drosophila melanogaster have been known for decades, as presented in figure 1 where the anterior (A), posterior (P), dorsal (D) and ventral (V) compartments are shown. Also, cells from the pouch (highlighted in figure) have a specific fate compared to non-pouch cells.

We present here the application of Coherent anti-Stokes Raman Scattering (CARS) spectromicroscopy to the study of cell patterns and local chemical composition in the wing imaginal disc without any additional label required. CARS is a third order non-linear optical process, ideal for non-invasively imaging of complex, e.g. biological, systems as it allows label-free, chemically specific, quantitative determination of the local concentration of the various species in a sample [1], with three dimensional submicron spatial resolution. As presented in figure 2, CARS allows collecting a quantitative vibrational spectrum at each position within the sample. This local information can be used to directly identify the shape and organization of cells across the boundaries between the distinct compartments of the disc by plotting the concentration of $\mathrm{CH}_{2}$ moieties within each voxel (Fig. 2, left). Simultaneously, by comparing the CARS spectra obtained in the different regions, direct comparison of the local chemical composition is readily available. This method enables to directly correlate the organization of the cells in the compartments of the imaginal wing disc to their chemical composition.

References

[1] M. Muller, and A. Zumbusch, ChemPhysChem. 8, 2156 (2007). 


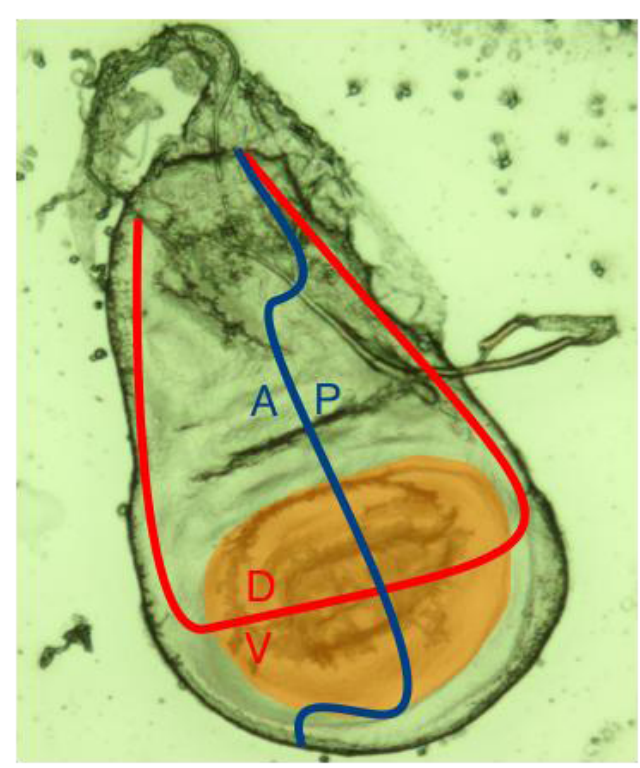

FIG. 1. Drosophila melanogaster wing imaginal disc. The vertical separation line between anterior (A) and posterior (P) compartment is shown in blue, whereas the separation between dorsal (D) and ventral (V) compartment is shown in red. The wing pouch is highlighted in orange.

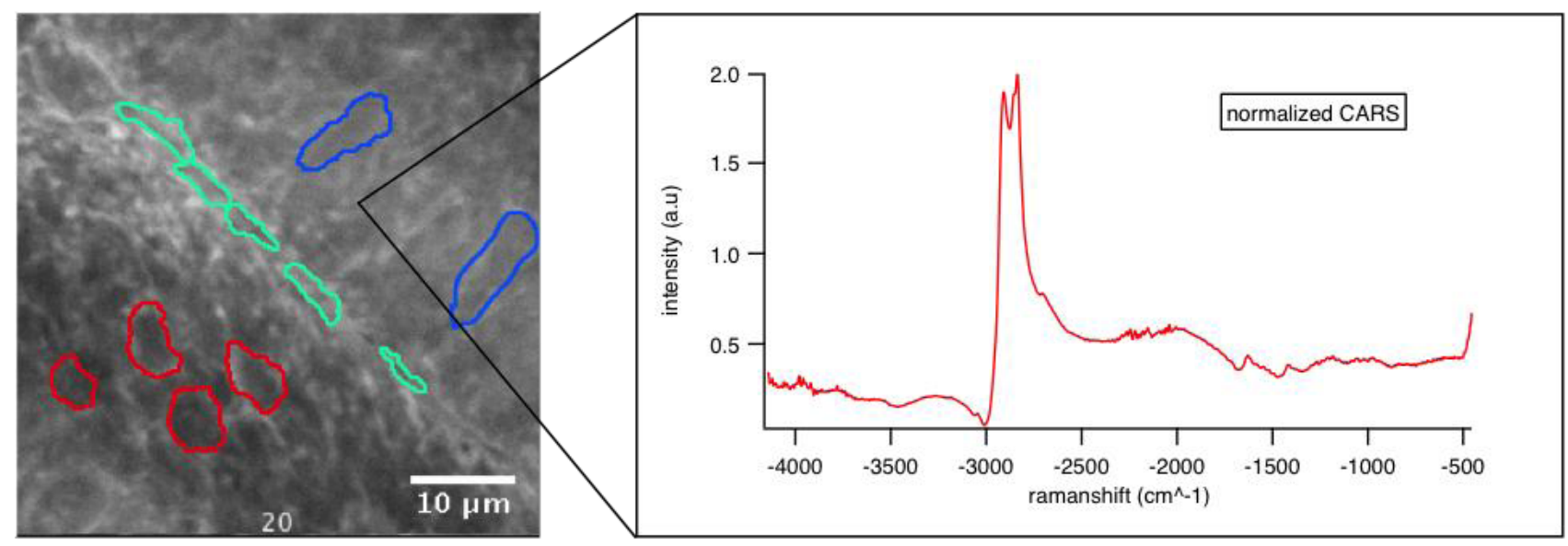

FIG. 2. CARS specto-microscopy of the imaginal wing disc. Right: the local concentration of $\mathrm{CH}_{2}$ vibrations provides an intrinsically confocal image of the organization of the cells. Left: at each voxel, a vibrational spectrum is acquired to determine the local chemical composition. 